Claremont Colleges

Scholarship@ Claremont

WM Keck Science Faculty Papers

W.M. Keck Science Department

4-29-2004

\title{
Behavior of Coupled Automata
}

Reuben Gann

Jessica Venable

Eric J. Friedman

Cornell University

Adam S. Landsberg

Claremont McKenna College; Pitzer College; Scripps College

\section{Recommended Citation}

Gann, R., J. Venable, E.J. Friedman, and A.S. Landsberg. "The Behavior of Coupled Automata." Physical Review E 69.4 (2004). DOI: 10.1103/PhysRevE.69.046116

This Article is brought to you for free and open access by the W.M. Keck Science Department at Scholarship @ Claremont. It has been accepted for inclusion in WM Keck Science Faculty Papers by an authorized administrator of Scholarship @ Claremont. For more information, please contact scholarship@cuc.claremont.edu. 


\title{
Behavior of coupled automata
}

\author{
Reuben Gann, ${ }^{1}$ Jessica Venable, ${ }^{1}$ Eric J. Friedman, ${ }^{2}$ and A. S. Landsberg ${ }^{1, *}$ \\ ${ }^{1}$ W. M. Keck Science Center, 925 N. Mills Avenue, Claremont McKenna, Pitzer, and Scripps Colleges, Claremont, California 91711, USA \\ ${ }^{2}$ School of Operations Research and Industrial Engineering, 206 Rhodes Hall, Cornell University, Ithaca, New York 14853, USA
}

(Received 29 April 2003; revised manuscript received 21 October 2003; published 29 April 2004)

\begin{abstract}
We study the nature of statistical correlations that develop between systems of interacting self-organized critical automata (sandpiles). Numerical and analytical findings are presented describing the emergence of "synchronization" between sandpiles and the dependency of this synchronization on factors such as variations in coupling strength, toppling rule probabilities, symmetric versus asymmetric coupling rules, and numbers of sandpiles.
\end{abstract}

DOI: 10.1103/PhysRevE.69.046116

PACS number(s): 05.65.+b, 45.70.-n, 05.45.-a

\section{INTRODUCTION}

Automata models displaying self-organized criticality (SOC) have received enormous critical attention over the last 15 years, and have been used as a paradigm for modeling statistical behaviors of a vast assortment of physical systems $[1,2]$. Given that physical systems are rarely found in complete isolation (e.g., consider a network of weakly interacting earthquake faults), it is quite natural to ask how one system (described by an SOC automaton model) might influence the behavior of a second automaton if the two are allowed to interact with one another. Despite our considerable knowledge to date about individual (isolated) SOC automata, systems of interacting SOC automata have been much less well studied, and comparatively little is known about the statistical effects associated with interactions. Recently, it has been demonstrated that if two two-dimensional "sandpile" automata are weakly coupled to one another, then the sandpiles will exhibit a remarkably high degree of synchronization in their avalanching behavior (cf., in any given avalanche, the root-mean-square (rms) fractional deviation of the avalanche size between the two sandpiles approaches zero regardless of the weakness of the coupling) [3]. This strong correlation only manifests itself on large spatial scales, and has been dubbed "large-scale synchrony." The intention of this paper is to examine the behavior of interacting automata more fully and in a wider range of contexts than has been done previously. Towards this end, we will examine statistical correlations in systems consisting of 2-20 coupled one-dimensional sandpile automata, and study how the onset of strong correlations/synchrony is affected by variations in coupling strength, dynamical toppling rules, number of automata, symmetric versus nonsymmetric coupling, and identicalness versus nonidenticalness of the individual automata.

This paper is organized as follows. Section II introduces the coupled sandpile models, and presents a broad survey of numerical results. Section III presents several basic analytical calculations for a two-sandpile system: a description of the underlying probabilities which define the structure of the SOC state, the relationship between the avalanching process

\footnotetext{
*Corresponding author.

Email address: landsberg@physics.claremont.edu
}

in sandpiles and random walks, and a calculation of the twopoint correlation functions for the system. These in turn provide the basis for the discussion and interpretation of our numerical findings in Sec. IV. Section V summarizes our key results, and discusses certain unresolved issues and limitations of the methodology we employed.

\section{SURVEY OF NUMERICAL RESULTS}

\section{A. Introduction to basic model}

Our study will focus on systems of coupled onedimensional directed automata (sandpiles) with stochastic toppling rules. The individual sandpiles are one-dimensional lattices (chains) of length $M$, where each lattice site $\{i$ $=1, \ldots, M\}$ contains some integral number of grains $h_{i}$. If the number of grains $h_{i}$ at any site exceeds a certain critical capacity $h_{c}$ (we take $h_{c}=4$ for convenience), then that site will topple, spilling one or more grains to its neighboring sites (either on its own chain or a neighboring chain) according to some probabilistic rules.

We focus for the moment on the operation of a single chain in isolation (which is solvable [5]). In this case, the specified toppling rule is as follows: An unstable lattice site (i.e., with $h_{i}>4$ ) will spill either one grain or two grains to its neighbor on its immediate right with probabilities $\rho_{1}, \rho_{2}$, respectively (where $\rho_{1}+\rho_{2}=1$ ). The original site will continue to shed grains through this toppling process until it eventually stabilizes $\left(h_{i} \leqslant 4\right)$. If the neighboring site to which the grains have been transferred becomes unstable as a result of this process, it in turn begins to topple, and the "avalanche" continues its rightwards march along the chain. The avalanche will either terminate on its own naturally or will stop when it reaches the right end of the chain (where it is assumed that any grains spilled from the rightmost lattice site simply drop off the chain). We define the total "size" $N$ of an avalanche to be the total number of grains spilled as the result of the addition of a single grain to the system.

To initially configure the sandpile, we start the lattice off in an arbitrary, quiescent state $\left(h_{i} \leqslant 4\right.$ for all $\left.i\right)$. A single grain is then added to a randomly selected site and the resulting avalanche (if any) is allowed to run its course. Once the system returns to a quiescent state, another site is selected at random for seeding. By repeatedly seeding the sandpile in this manner, transient behaviors are eliminated 


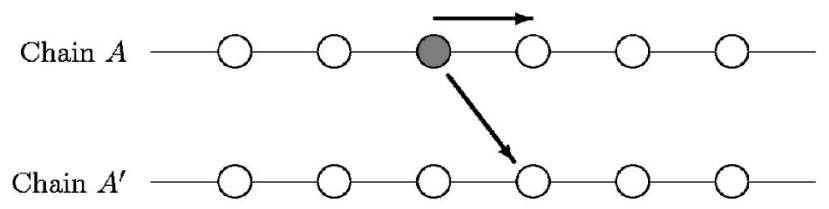

FIG. 1. Depiction of two interacting lattices. The arrows indicate the possible spilling directions for an above-critical site (represented by a filled circle).

and the system enters the SOC state. In all discussions that follow it should be assumed that this preliminary seeding process has been carried out. If additional grains are now added to the sandpile, the frequency of avalanches as a function of their size exhibits the familiar power-law scaling behavior of SOC [6], $F(N) \sim 1 / N^{d}$. For the case of a single isolated sandpile, the critical exponent is $d=4 / 3$ (independent of the spill probabilities $\rho_{1}, \rho_{2}$, provided $\left.\rho_{1} \neq 0,1\right)$. The behavior of this type of one-dimensional SOC sandpile model appears sufficiently general [8] to serve as a good basis for a study of interacting SOC automata, as we now describe.

Systems of interacting automata may be constructed by coupling together two or more of the basic one-dimensional sandpiles described above. Coupling is achieved by modifying the toppling rules so that a site on a given sandpile is capable of spilling grains not only to a neighbor on its own chain, but also to neighboring sites on nearby chains as well. We consider first the case of two interacting sandpiles: Take two one-dimensional lattices, each of length $M$, and place them side by side, as shown in Fig. 1. (Call these chains $A$ and $A^{\prime}$.) As in the previous case of a single isolated sandpile, assume all sites are stable provided they contain no more than $h_{c}=4$ grains. The toppling rules are as follows: If a site $i$ on chain $A$ topples, it can spill $\alpha$ grains to site $i+1$ on $A$ and $\beta$ grains to site $i+1$ on $A^{\prime}$ with probability $\rho_{\alpha \beta}$. the analogous spill probabilities for a toppling site on $A^{\prime}$ are denoted as $\rho_{\alpha \beta}^{\prime}$. For simplicity we assume that $\rho_{10}, \rho_{20}, \rho_{11}, \rho_{22}, \rho_{10}^{\prime}, \rho_{20}^{\prime}, \rho_{11}^{\prime}, \rho_{22}^{\prime}$ are the only nonzero spill probabilities, although this will not affect the generality of our results.

When $\rho_{\alpha \beta}=\rho_{\alpha \beta}^{\prime}$ for all $\alpha, \beta$, the two sandpiles are governed by the same probabilistic rules and we will refer to this as the symmetric case. Lastly, we will often work with a special case of the above toppling rule, whereupon we introduce four free parameters $\rho, \gamma$ and $\rho^{\prime}, \gamma^{\prime}$ [all of which lie in the range $(0,1)]$, and demand that our eight toppling probabilities $\rho_{\alpha \beta}, \rho_{\alpha \beta}^{\prime}$ are of the special form $\rho_{10}=\rho(1-\gamma), \rho_{20}$ $=(1-\rho)(1-\gamma), \rho_{11}=\rho \gamma, \rho_{22}=(1-\rho) \gamma$, and similarly for the primed variables. The parameters $\rho, \gamma$ and $\rho^{\prime}, \gamma^{\prime}$ lend themselves to a simple interpretation: $\gamma$ is the probability that spilling grains from a toppling site on chain $A$ will cross over and hit the other chain $A^{\prime}$ (hence $1-\gamma$ is the probability that the spill does not cross over). $\rho$ is a probability associated with one-grain spills on $A ; 1-\rho$ is associated with two-grain spills on $A$. The quantities $\rho$ and $\gamma$ should be viewed as independent. So, for example, the probability that when a site on $A$ topples it spills one grain to its own chain and none to the other is $\rho(1-\gamma)$, while the probability that it spills one grain to each is $\rho \gamma$. (The parameters $\rho^{\prime}, \gamma^{\prime}$ are the analogous quantities defined for when the toppling site is on chain $A^{\prime}$.) The virtue of this formulation is that the parameters $\gamma, \gamma^{\prime}$ provide convenient measures of the coupling strength between the two sandpiles, since if $\gamma=\gamma^{\prime}=0$ the sandpiles are dynamically independent of one another, while if $\gamma=\gamma^{\prime}=1$, then they are "fully coupled" (in that whenever any site topples, it spills the same number of grains to each sandpile.) By choosing $\gamma \neq \gamma^{\prime}$ we can explore the case of asymmetric coupling as well. We mention that while the special case we are considering here for the toppling rule (based on four independent parameters $\left.\rho, \gamma, \rho^{\prime}, \gamma^{\prime}\right)$ is more restrictive than our original formulation (which employs six independent parameters), it is only nominally so, in that the overall methodology is easily generalizable. (In fact, while most of our numerical simulations will employ $\rho, \rho^{\prime}, \gamma, \gamma^{\prime}$ for convenience, all of our analytical calculations will use the more general $\rho_{\alpha \beta}, \rho_{\alpha \beta}^{\prime}$ formulation.)

We now turn to numerical observations about the statistical behavior of interacting automata.

\section{B. Synchronization in a two-chain system with weak, symmetric coupling}

As a prelude to other results which follow, we review the basic synchronization behavior of two sandpiles which are weakly coupled to one another in a symmetric fashion $\left(\gamma=\gamma^{\prime} \ll 1\right)$ as studied for a deterministic model in Ref. [3]. Grains are dropped one at a time onto randomly selected sites on either sandpile, and the resulting avalanches are monitored. For each drop, we record the size $N$ of the resulting avalanche (i.e., the total number of grains spilled), as well as the individual contributions to this total coming from each of the two chains $N_{A}, N_{A^{\prime}}$ (where $\left.N_{A}+N_{A^{\prime}}=N\right)$. A representative plot of $N_{A}$ vs $N_{A^{\prime}}$ is shown in Fig. 2. The high concentration of data points along the $N_{A}$ and $N_{A^{\prime}}$ axes for small avalanche sizes indicates that small avalanches on each sandpile are essentially uncorrelated with one another. For larger avalanches, the correlation in the avalanching between the two sandpiles not only becomes stronger (as one might also naturally expect), but in fact becomes so strong that a large event on one sandpile is almost always concomitant with an approximately equal size event on the other sandpile, as evidenced by the pronounced tendency towards the diagonal in the graph at large scales. The graph thus depicts the emergence of the phenomenon of large-scale synchrony for large avalanches (i.e., $N_{A} \approx N_{A^{\prime}}$ ). (We remark that this is not a saturation effect associated with finite lattice size.)

Note that reducing the strength of the coupling $(\gamma)$ between the sandpiles does not destroy the large-scale synchrony, but merely delays its onset (as can be analytically demonstrated through a dynamical renormalization analysis as described in Ref. [3]). What happens is that even though on a microscopic level the two chains are only weakly coupled [4] via the local toppling rules $(0<\gamma \ll 1)$, a renormalization analysis shows that the effective coupling strength between the sandpiles increases as one views them on larger and larger spatial scales, thereby producing the observed synchrony. 


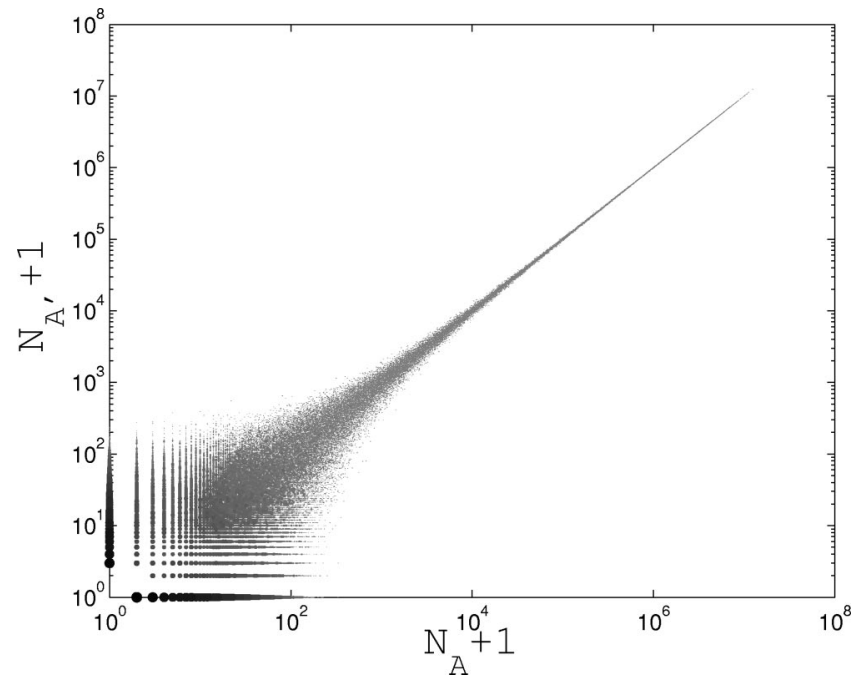

FIG. 2. Large-scale synchrony. Plot illustrates dependence of avalanche composition on size in a two-chain model for $\gamma=\gamma^{\prime}=0.05, \rho=\rho^{\prime}=0.5$. Note in particular the strong correlations (i.e., $N_{A} \approx N_{A^{\prime}}$ ) for large avalanches despite the weak coupling; the much more frequent smaller avalanches are relatively uncorrelated. Typical lattice sizes used in the simulations ranged from 50000 to 200000.

Numerically, one can examine the root-mean-square fractional deviation between $N_{A}$ and $N_{A^{\prime}}$ as a function of total avalanche size $N$,

$$
f_{\mathrm{rms}}=\sqrt{\left\langle\frac{\left(N_{A}-N_{A^{\prime}}\right)^{2}}{\left(N_{A}+N_{A^{\prime}}\right)^{2}}\right\rangle},
$$

where the brackets $\langle\cdot\rangle$ indicate an average over all avalanches of a given total size [9]. Figure 3 clearly depicts how uncorrelated small avalanches $\left(f_{\text {rms }} \approx 1\right)$ give way to statisti-

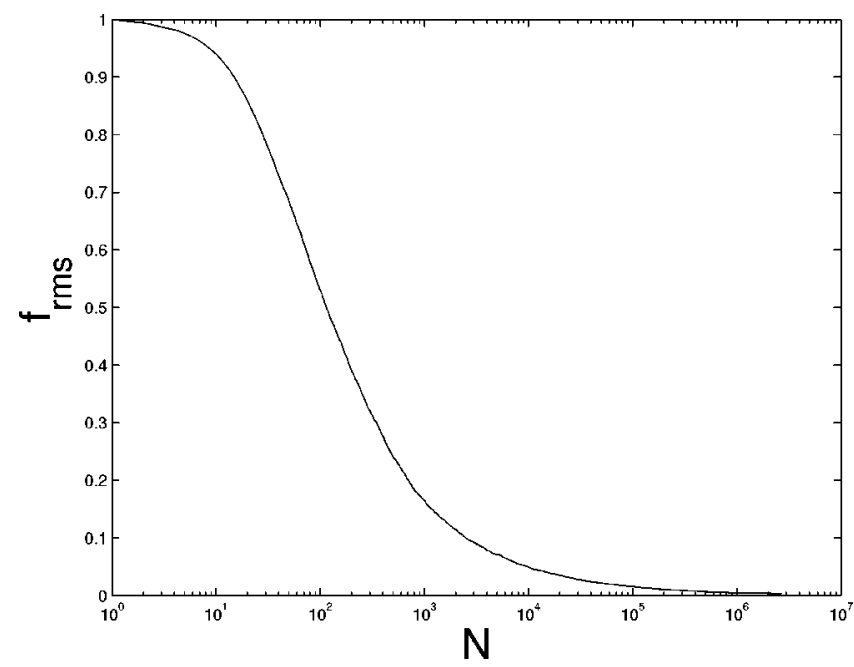

FIG. 3. The root-mean-square fractional deviation between $N_{A}$ and $N_{A^{\prime}}$ vs the total avalanche size $\left(N=N_{A}+N_{A^{\prime}}\right)$, for $\gamma=\gamma^{\prime}=0.05, \rho=\rho^{\prime}=0.5$. Note that the fractional deviation approaches zero at large avalanche size, corresponding to the development of the strong correlations seen in Fig. 2.

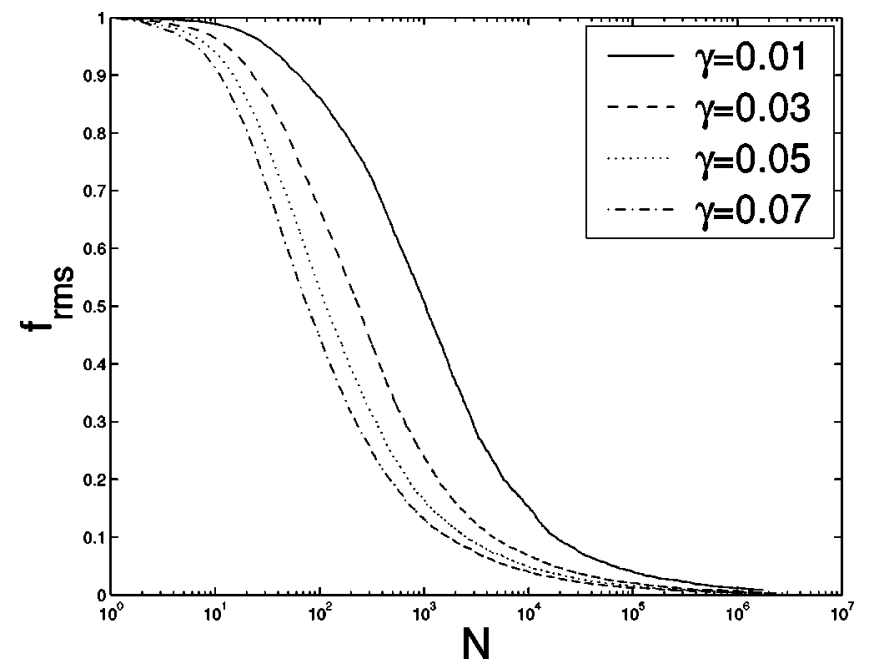

FIG. 4. The effect of coupling strength on $f_{\text {rms }}$, in the symmetric case with $\gamma=\gamma^{\prime}, \rho=\rho^{\prime}=0.5$. As the coupling between sandpiles is reduced, the onset of large-scale synchrony between sandpiles is pushed back to progressively larger length scales.

cally synchronous behavior $\left(f_{\mathrm{rms}} \approx 0\right)$ at large spatial scales. (We point out that since the generation of reliable statistical data for very large avalanche sizes is a numerically taxing process, it proved useful to employ a binning procedure of the data when generating $f_{\text {rms }}$ plots like the one shown. In the figure, avalanches between size $n$ and $2 n$ were binned together.) Note that the rms fractional deviation is chosen for simplicity; other more sophisticated measures of correlation could also serve this purpose, and might be interesting to study.

We now turn to our main focus, an examination of how such statistical correlations between automata are affected by various systematic changes, e.g., modifications to toppling rules, system parameters, boundary conditions, number of automata, etc.

\section{Coupling strength effects in a symmetric two-chain system}

If the coupling strength in a symmetric two-chain system (above) is varied, the onset of large-scale statistical synchrony will be affected. Figure 4 illustrates this relationship in terms of the associated $f_{\text {rms }}$ plots; a delay in onset of synchrony as the coupling is reduced is clearly seen. To quantitatively study this dependency we choose some threshold $f_{\text {onset }}$, and define the onset of large-scale synchrony to be when the $f_{\text {rms }}$ curve drops below this cutoff. In the discussion which follows we use $f_{\text {onset }}=0.5$ unless otherwise indicated. A plot of avalanche size at onset $N_{\text {onset }}$ as a function coupling strength $\gamma$ is shown in Fig. 5(a). As noted, decreasing the coupling strength pushes back the onset of synchronization to progressively larger length scales. The asymptotic shape of this curve in the weak-coupling regime is shown in Fig. 5(b). The curve (at least superficially) appears to be fit relatively well by a power law: $N_{\text {onset }} \sim \gamma^{-q}$, where $q=1.4$ \pm 0.1 . This point proves to be somewhat subtle and we will return to this issue in more detail in the analytical discussion of Sec. IV. We mention here that exploring this weak- 

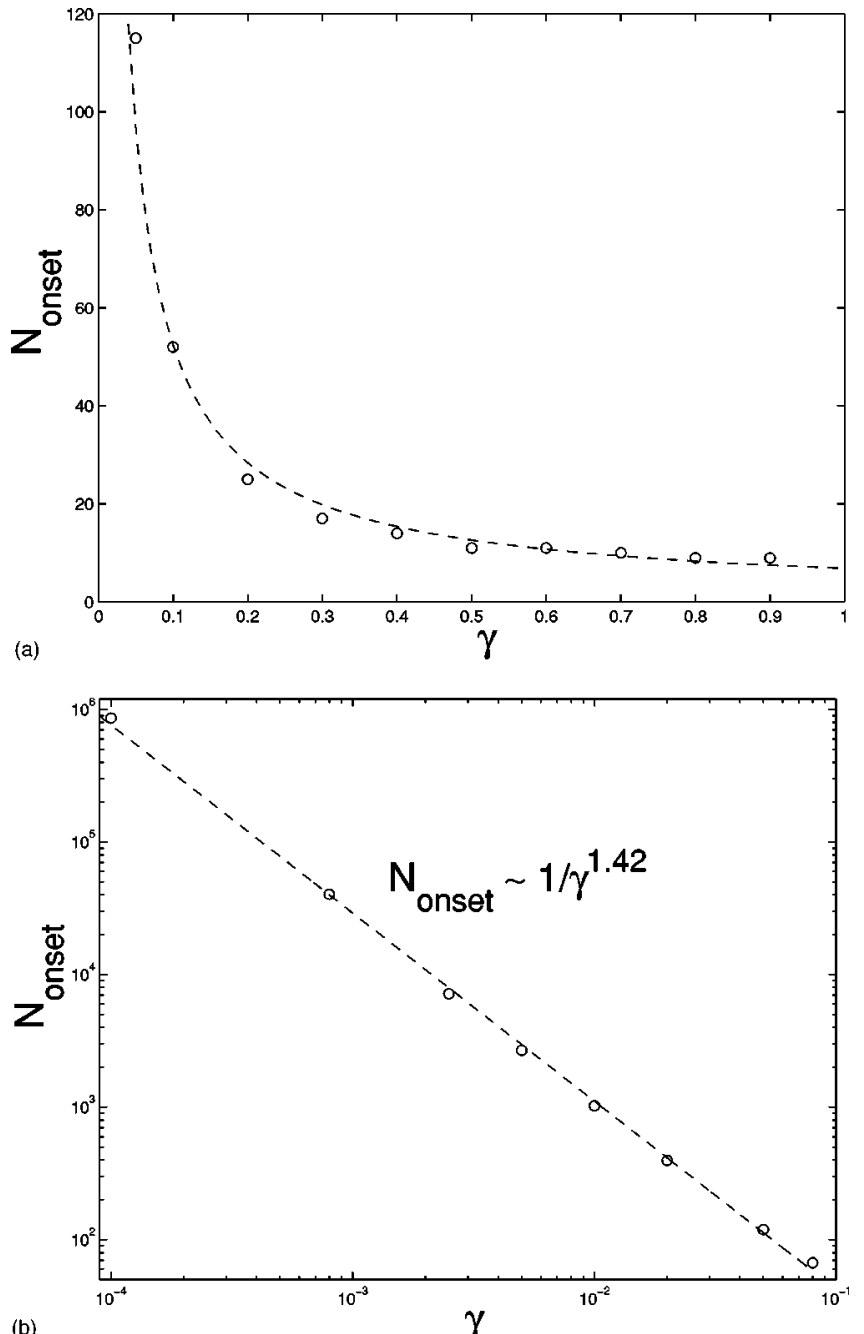

(b) $\gamma$

FIG. 5. (a) Onset of synchronization as a function of coupling strength for the symmetric two-chain case with $\rho=\rho^{\prime}=0.5$, (b) Blow-up of graph in weak-coupling regime (on a log-log scale). A numerical power-law fit is shown.

coupling regime is a numerically intensive process, since synchronization only sets in at very large avalanche size, and large avalanches occur only infrequently. Yet good statistical data for the large avalanches is precisely what is required for a reliable determination of $f_{\mathrm{rms}}$ and $N_{\text {onset }}$. Additionally, as one decreases the coupling strength, the lattice sizes used in the simulations (to accommodate the increasingly large avalanches) must also be increased to ensure that boundary effects do not distort the results [7].

\section{Synchrony in an asymmetrically coupled two-chain system}

We next consider the system's behavior when the coupling between the two sandpiles is not symmetric, $\gamma \neq \gamma^{\prime}$. For convenience we still assume $\rho=\rho^{\prime}$ (this proves to be a rather mild restriction, and in the analysis of Sec. III we take up the general case where $\rho \neq \rho^{\prime}$ ). Our numerical findings for asymmetric coupling are illustrated in Fig. 6. Figure 6(a) shows the $f_{\text {rms }}$ curves for one set of coupling-strength values. Observe that while the $f_{\text {rms }}$ curves tend to decrease with increas-
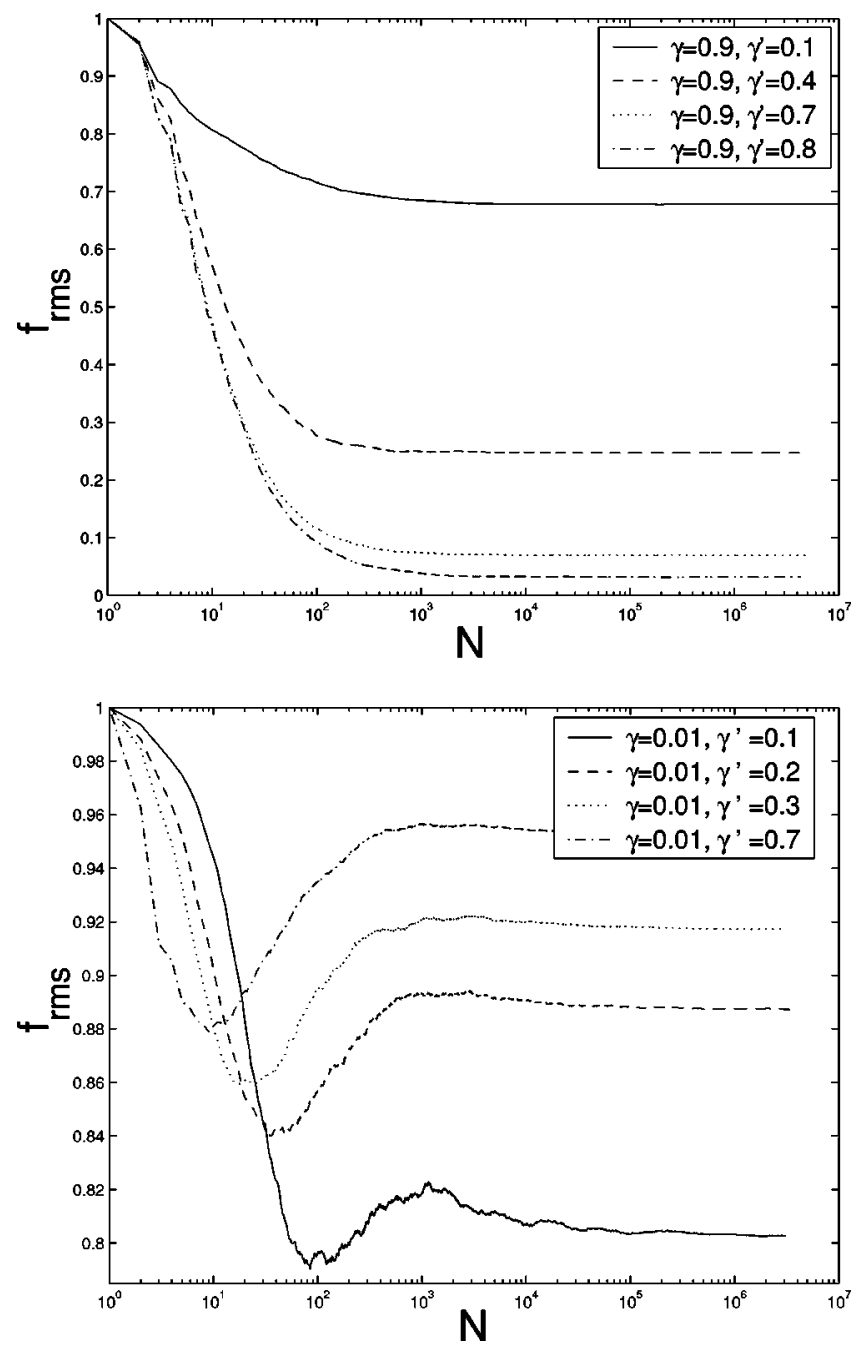

FIG. 6. (a) Plateauing rms curves for systems with $\rho=\rho^{\prime}=0.5$ for the asymmetric case $\left(\gamma \neq \gamma^{\prime}\right)$. Note that the plateauing $f_{\text {rms }}$ value increases as $\left|\gamma-\gamma^{\prime}\right|$ increases, (b) Same as in (a), but for different choices of coupling parameters $\gamma \neq \gamma^{\prime}, \rho=\rho^{\prime}=0.5$. In addition to the asymptotic plateauing behavior already noted, local minima (dips) in the $f_{\text {rms }}$ curves are clearly visible in the intermediate range of $N$.

ing avalanche size, they no longer asymptotically approach zero at large spatial scales. Rather, they each eventually plateau at some value between 0 and 1, indicating that during large avalanches more grains are spilled from one chain than the other. Qualitatively this is as expected, since the unequal coupling strengths imply that one chain is more "generous" with its spilled grains than the other. Figure 6(b) depicts the $f_{\text {rms }}$ curves for a different set of coupling-strength values. Here, we again see the eventual plateauing behavior at large scales, but now a pronounced dip with subsequent rebound is clearly visible before the plateau is reached. (A simple explanation for this numerical result is described in Sec. IV B.)

\section{E. Sandgrain distribution}

We consider again the case of a symmetrically coupled two-chain system, and examine the distribution of sandgrains in the SOC state. Letting $F_{i}$ denote the fraction of sites containing $i$ grains $(i=1, \ldots, 4)$, Table I below illustrates some 
TABLE I. Depicts the fraction of lattice sites containing 4, 3, 2, and 1 grain, respectively. Note the good agreement between the theoretically computed values and the simulation results across a broad range of coupling-strength values.

\begin{tabular}{lcccccccc}
\hline \hline Chain parameters & $F_{4}^{\text {numeric }}$ & $F_{4}^{\text {theory }}$ & $F_{3}^{\text {numeric }}$ & $F_{3}^{\text {theory }}$ & $F_{2}^{\text {numeric }}$ & $F_{2}^{\text {theory }}$ & $F_{1}^{\text {numeric }}$ & $F_{1}^{\text {theory }}$ \\
\hline$\gamma=0.0$ & 0.669 & 0.667 & 0.332 & 0.333 & 0 & 0 & 0 & 0 \\
$\gamma=0.04$ & 0.641 & 0.641 & 0.333 & 0.333 & 0.0125 & 0.0128 & 0.0136 & 0.0128 \\
$\gamma=0.07$ & 0.622 & 0.623 & 0.334 & 0.333 & 0.0233 & 0.0218 & 0.0212 & 0.0218 \\
$\gamma=0.7$ & 0.396 & 0.392 & 0.329 & 0.333 & 0.137 & 0.137 & 0.138 & 0.137 \\
\hline \hline
\end{tabular}

sample numerical results for several different values of the coupling-strength parameter $\gamma$ (at fixed $\rho=0.5$ ). Numerical values given are reproducible to an accuracy of $\approx \pm 0.004$. (The theoretical predicted values appearing in the table will be discussed in Sec. III B.)

\section{F. Multichain systems, site restrictions, and related observations}

We have also studied several other generalizations which we briefly describe here. More details on these special cases can be obtained directly from the authors; all can be analyzed using the same general framework developed in this paper.

We first mention "site-restricted: automata, which are identical to those studied here except that now only some fraction $r<1$ of the lattice sites are capable of spilling grains to the other chain; the remainder can only spill to neighboring sites on their own chain. The sites capable of cross spills can be periodically or randomly located along each chain. The motivation for studying site restrictions comes from considering spatially distributed SOC systems which happen to only be linked at a few select sites, as might naturally be expected to occur in real physical systems. Numerical observations show that strong correlations between site-restricted automata develop at large scales even for the case of severe site restriction $(r \ll 1)$, a result which might be anticipated in light of the analysis to be presented in Secs. III and IV. For such systems, the effective coupling strength between the automata is determined by the quantity $r \gamma$.

We also considered the statistical correlations between three interacting sandpiles (non-site-restricted) where the top and bottom chains can spill grains directly to the center chain, though not to each other. The center chain can spill to either the top or bottom chain. In this case numerical simulations show that the chains synchronize, with the middle chain having twice the number of events as either the top or bottom chain, due to the effect of the middle chain having twice the linkages of the top (or bottom) chain.

Lastly, we simulated models with 10 and 20 chains with symmetric, nearest-neighbor coupling between chains for both periodic and nonperiodic boundary conditions. As expected from general considerations to be described later, large-scale synchrony was observed in all these systems, with the synchrony between any two chains setting in at progressively larger avalanche sizes the farther the two chains were from each other.

\section{ANALYTICAL CALCULATIONS AND RELATED RESULTS}

We next present several analytical results along with plausibility arguments that will prove useful for understanding the behavior of a system of two interacting sandpiles. We note that the formal calculations all readily generalize to higher-dimensional systems of interacting sandpiles (of similar type), in support of our earlier contention that the onedimensional automata that we study here are sufficient to capture many of the important features of higherdimensional interacting automata. These results will then be used in Sec. IV to interpret many of our earlier numerical findings.

\section{A. Spill probability calculation}

In this section we calculate spill probabilities associated with the critical sites $\left(h_{i}=4\right)$ of the two-sandpile model of Sec. II A. This in turn will provide us with a description of the underlying structure of the SOC state of the interacting automata.

Let $w_{k}$ be the probability that if we drop $k$ grains onto a critical site on chain $A$ it will spill out exactly $k$ grains, while $x_{k}, y_{k}$, and $z_{k}$ are defined as the probabilities that the site will spill exactly $k+1, k+2$, or $k+3$ grains, respectively. [Equivalently, $w_{k}$ is the probability that a critical site will return to criticality following the addition of $k$ grains; $x_{k}$ is the probability that it will end up one grain below criticality (at $h_{i}=3$ ), $y_{k}$ that it ends at two below criticality, and $z_{k}$ at three below criticality.] This accounting is sufficient, since, for the models we are considering, a site that is $k$ grains above criticality will always spill at least $k$, and at most $k$ +3 , grains. We define spill probabilities $w_{k}^{\prime}, x_{k}^{\prime}, y_{k}^{\prime}, z_{k}^{\prime}$ for critical sites on chain $A^{\prime}$ analogously. Our goal is to calculate these spill probabilities in terms of the underlying toppling rule probabilities $\rho_{10}, \rho_{20}, \rho_{11}, \rho_{22}, \rho_{10}^{\prime}, \rho_{20}^{\prime}, \rho_{11}^{\prime}, \rho_{22}^{\prime}$.

We start with the observation that adding $k$ grains to a critical site is equivalent to first adding $k-1$ grains to the site and then adding one additional grain. Thus, for example, the probability that the addition of $k$ grains to a critical site results in exactly $k$ grains spilled out is $w_{k}=w_{k-1} \rho_{10}$ $+x_{k-1}$. Continuing this procedure yields: $x_{k}=w_{k-1}\left(\rho_{20}\right.$ $\left.+\rho_{11}\right)+y_{k-1}, y_{k}=z_{k-1}, z_{k}=w_{k-1} \rho_{22}$. (The corresponding recursion relations for critical states on chain $A^{\prime}$ are generated analogously.) Note that $w_{k}+x_{k}+y_{k}+z_{k}=1$ for all $k$ and that the "initial conditions" for these relations are $w_{1}$ $=\rho_{10}, x_{1}=\rho_{20}+\rho_{11}, y_{1}=0, z_{1}=\rho_{22}$. Since these recursion 
relations are linear, they are readily solvable and thus exact expressions for $\left(w_{k}, x_{k}, y_{k}, z_{k}\right)$ in terms of the initial conditions can be found. However, we are primarily interested in the large- $k$ limit (corresponding to the SOC regime in which large avalanches exist). For our purposes then, it suffices to note that if the recursion relations are reexpressed in matrix notation, then the associated recursion matrix has one eigenvalue equal to unity; its three other eigenvalues all have modulus less than unity, and hence are unimportant in the asymptotic regime $k \rightarrow \infty$. Consequently, the solutions $\left(w_{k}, x_{k}, y_{k}, z_{k}\right)$ become independent of $k$ for large $k$. We find

$$
\left(w_{k}, x_{k}, y_{k}, z_{k}\right) \approx \frac{1}{2-\rho_{10}+2 \rho_{22}}\left(1,1-\rho_{10}, \rho_{22}, \rho_{22}\right)
$$

(This approximate equality becomes exact in the limit $k \rightarrow \infty$; we denote the limiting values as $w_{\infty}, x_{\infty}, y_{\infty}, z_{\infty}$.) These are the spill probabilities for the critical sites of the automata upon the addition of $k$ grains. As described next, this result will prove useful for understanding the structure of the SOC state.

\section{B. Grain distribution in the SOC state}

We show here how result (2) above can be used to extract information about the underlying structure of the SOC state of the system. Recall first that the spill probabilities $\left(w_{k}, x_{k}, y_{k}, z_{k}\right)$ were originally defined solely in terms of how a critical site (at $\left.h_{i}=4\right)$ would respond to the addition of $k$ grains: $w_{k}$ was the probability that it would return to criticality, $x_{k}$ that it would end up one below criticality (at $h_{i}=3$ ), etc. However, since these probabilities are independent of $k$ for large $k$, these results also hold for any site $\left(h_{i}\right.$ $=1,2,3$, or 4) -not just for critical sites. To see this, consider a site that is initially $m$ grains below criticality, at $h_{i}$ $=4-m$. If $k$ grains are added to it, the first $m$ grains will simply bring the site up to criticality. So when the remaining $k-m$ grains are added, the probability that the now critical site will return to criticality when it sheds the excess is $w_{k-m}$. However, since $w_{k-m} \approx w_{k}$ for large $k, w_{k}$ in fact describes the probability that an arbitrary site (initially at or below criticality) will end up being critical after a large number of grains are added to it, as claimed. In turn, it follows that if the automata is in the SOC state, we can interpret the asymptotic spill probability $w_{\infty}$ in result (2) to be the probability that a randomly selected site on chain $A$ will be critical, while $x_{\infty}, y_{\infty}, z_{\infty}$ give the probabilities that a site on the chain contains 3, 2, or 1 grains, respectively. Thus the underlying distribution of states of the automata is fully revealed. A comparison between the predicted fraction of critical sites and numerical simulations is given in Table I. (Note that minor discrepancies between theory and numerics are expected owing to finite lattice size and finite sample size.)

In addition, we note that due to the directed nature of the system the grain distribution is independent between sites. This can be shown formally by constructing the Markov chain representing the grain size distribution at all sites and showing that the invariant distribution for the entire system can be written as a product of the invariant distributions of the individual sites. Note however that this does not prove that successive avalanches are uncorrelated, something which is generally not true for deterministic SOC systems, although it might appear plausible for this specific case.

\section{Connection to random walks}

We next indicate how avalanching in a single onedimensional directed sandpile can readily be interpreted as a stochastic process. Given a drop (of a single grain) onto some site let $X_{t} \geqslant 0$ be the random variable representing the number of grains spilled by the site $t$ steps away from the drop site. Note that the infinite sequence $X$ $=\left(X_{1}, X_{2}, X_{3}, \ldots\right)$ can be viewed as a random walk with 0 as its absorbing state, since if $X_{t}=0$ then $X_{s}=0$ for all $s$ $>t$. By the analysis in Sec. III A $\left|X_{t}-X_{t+1}\right| \leqslant 3$, and, because of grain conservation, the expected number of spills at a site conditional on the number of spills at the previous site is given by, $E\left[X_{t+1} \mid X_{t}\right]=X_{t}$ (where $E[\cdot \mid \cdot]$ denotes the conditional expectation), so the sequence corresponds to a fair random walk. [Note that one can show this directly by combining the analysis in Sec. III A with that in the preceding section, by explicitly computing $E\left[X_{t+1} \mid X_{t}=j\right]$ $=\sum_{k=1}^{4} E\left[X_{t+1} \mid X_{t}, h_{t+1}=k\right] \operatorname{Pr}\left(h_{t+1}=k\right)=j+\left(-3 w_{j-3}\right.$ $\left.-2 x_{j-3}-1 y_{j-3}-0 z_{j-3}\right) z_{\infty}+\left(-2 w_{j-2}-1 x_{j-2}-0 y_{j-2}\right.$ $\left.+1 z_{j-2}\right) y_{\infty}+\left(-1 w_{j-1}+0 x_{j-1}+1 y_{j-1}+2 z_{j-1}\right) x_{\infty}+\left(0 w_{j}\right.$ $\left.+1 x_{j}+2 y_{j}+3 z_{j}\right) w_{\infty}$.] Hence, for large values of $X_{t}$ (as arise in large avalanches) this sequence can be approximated by a Brownian motion [15].

We would like later to exploit this direct association between avalanching behavior in a single sandpile and Brownian motion. Unfortunately, for the coupled sandpile case this connection to Brownian motion breaks down. However, as we will see later, ideas from renormalization theory allow us to apply these ideas to understand multichain systems.

\section{Two-point correlation functions}

In this section we derive our final analytical result, the two-point correlation functions for our system of interacting sandpiles. These correlation functions describe the expected number of times a given site will topple as a result of adding a single grain to some other specified site of the system. More precisely, suppose we drop a single grain onto a site on chain $A$. Then $P(i, A)$ is defined to be the expected number of times that the lattice site on $A$ located exactly $i$ units to the right of the initial drop site will topple; $P\left(i, A^{\prime}\right)$ is defined as the expected number of times that the corresponding site on $A^{\prime}$ located $i$ units from the initial drop site will topple. Analogously, if instead a single grain is added to a site on chain $A^{\prime}$, then the correlation function $P^{\prime}\left(i, A^{\prime}\right)$ [resp. $\left.P^{\prime}(i, A)\right]$ denotes the expected number of topplings induced at a site $i$ units away on chain $A^{\prime}$ (resp. $A$ ). [Note: by definition, the correlation functions describe the expected number of times a site topples, not the total number of grains spilled from the site. Recall that a site can spill between one and four grains each time it topples, as described in Sec. II A.] 
The correlation functions may be derived from the general observation (as described by Dhar [13]) that, for automata in the SOC state, the average rate at which grains enter any given site must equal the average rate at which they leave (see also [14]). A straightforward calculation (as in Ref. [3]) yields

$$
P(i, A)=c_{1}+c_{2} \lambda^{i}, \quad P\left(i, A^{\prime}\right)=c_{3}-c_{3} \lambda^{i},
$$

where $c_{1}, c_{2}, c_{3}, \lambda$ are constants which are readily determined. (For symmetric sandpiles, $c_{1}, c_{2}, c_{3}$ are all equal.) Since $|\lambda|<1$ (except for trivial cases), the limiting behavior of the correlation functions far from the drop site $(i \gg 1)$ is transparent. $\left[P^{\prime}\left(i, A^{\prime}\right), P^{\prime}(i, A)\right.$ are found similarly. $]$

\section{DISCUSSION OF NUMERICAL FINDINGS}

We now use the analytical results of Sec. III to interpret many of the original numerical findings of Sec. II. For example, we will be able to explain the plateaus and dips seen in the $f_{\text {rms }}$ curves for nonsymmetric sandpiles, and the observed dependence of the onset of synchronization on intersandpile coupling strength.

\section{A. Basic synchronization}

First we note that one might anticipate the emergence of large-scale synchrony between coupled sandpiles as illustrated in Figs. 2 and 3 of Sec. II B from the two-point correlation functions (as in Ref. [3]). Observe that for $i \gg 1$ and symmetric coupling, $P(i, A) \approx P\left(i, A^{\prime}\right) \approx P^{\prime}(i, A)$ $\approx P^{\prime}\left(i, A^{\prime}\right)$. This implies that dropping a grain at some site affects distance sites on either chain equally strongly (on average); i.e., as the avalanche propagates down a chain, memory of whether the initial drop was onto chain $A$ or chain $A^{\prime}$ is lost. This is constant with (and suggestive of) the observation of large-scale synchrony between sandpiles wherein the rms-fractional deviation drops towards zero (Fig. 3), though it does not prove it. (A stronger argument would utilize a dynamical renormalization group analysis, as in Ref. [3].)

\section{B. Plateauing in asymmetric sandpiles}

The key numerical finding of Sec. II D is that if two sandpiles are asymmetrically coupled, then the system only partially synchronizes, as evidenced by the plateaus observed at large length scales in the associated $f_{\text {rms }}$ plots [Fig. 6(a)]. This behavior can be well explained using the two-point correlation functions, as we now describe.

For simplicity we work with the auxiliary parameters $\rho, \gamma, \rho^{\prime}, \gamma^{\prime}$ (see Sec. II A) and define $n_{A A^{\prime}} \equiv(2-\rho) \gamma, n_{A^{\prime} A}$ $\equiv\left(2-\rho^{\prime}\right) \gamma^{\prime}, \quad n_{A} \equiv(2-\rho)(1+\gamma), \quad$ and $\quad n_{A^{\prime}} \equiv\left(2-\rho^{\prime}\right)(1$ $\left.+\gamma^{\prime}\right)$.

To begin, suppose a single grain is initially dropped onto some site on chain $A$. (We will assume that for these sandpiles $\rho_{10}=\rho_{10}^{\prime}, \rho_{20}=\rho_{20}^{\prime}, \rho_{11} \neq \rho_{11}^{\prime}, \rho_{22} \neq \rho_{22}^{\prime}$.) The expected number of topplings this initial drop will induce at some distant site on chain $A$ is, according to Eq. (3), $\approx c_{1}$. Since each time a site on $A$ topples it spills an average of $n_{A}$ grains
TABLE II. Depicts asymptotic value of the rms fractional deviation for different realizations of the asymmetric intersandpile coupling. Note the good agreement between the numerically computed and predicted values.

\begin{tabular}{lcc}
\hline \hline Values of $\gamma$ & $f_{\text {rms }}^{\text {asymptote }}$ Theory & Simulation Result \\
\hline$\gamma=0.9, \gamma^{\prime}=0.1$ & 0.67797 & 0.67770 \\
$\gamma=0.9, \gamma^{\prime}=0.5$ & 0.17391 & 0.17363 \\
$\gamma=0.5, \gamma^{\prime}=0.7$ & 0.10526 & 0.10517 \\
$\gamma=0.0, \gamma^{\prime}=0.07$ & 1.00000 & 0.99995 \\
$\gamma=0.04, \gamma^{\prime}=0.7$ & 0.82914 & 0.82874 \\
\hline \hline
\end{tabular}

(as is easily checked), the average flux of grains emerging from the distant site is $c_{1} n_{A}$. Likewise, the average flux out of a distant site on chain $A^{\prime}$ is $c_{3} n_{A^{\prime}}$. (As an aside, we note that the average rate at which particles are transferred from a given distant site on $A$ to $A^{\prime}$ is equal to the average transfer rate at distant sites from $A^{\prime}$ to $A$, a finding consistent with the existence of large-scale synchrony between the sandpiles.) Continuing, the key observation is that the ratio of the average flux out of a distant site on $A$ to the flux out of a distant site on $A^{\prime}$, namely $c_{1} n_{A} / c_{3} n_{A^{\prime}}$, is equal to the ratio of the total number of grains spilled by sites on $A$ to the number spilled by sites on $A^{\prime}$ during a given avalanche, namely $N_{A} / N_{A^{\prime}}$. Using this fact along with the readily computed values of $c_{1}, c_{3}$ in Eq. (3), the rms fractional deviation $f_{\text {rms }}$ given by Eq. (1) may be reexpressed as

$$
f_{\text {rms }}^{\text {asymptote }}=\left|\frac{n_{A^{\prime}{ }_{A} n_{A}-n_{A A^{\prime}} n_{A^{\prime}}}}{n_{A^{\prime}{ }_{A} n_{A}+n_{A A^{\prime}} n_{A^{\prime}}}}\right| .
$$

This result gives the expected plateau levels seen in $f_{\text {rms }}$ plots [e.g., Figs. 6(a) and 6(b)].

Table II compares the theoretical predicted and observed values of the plateaus for $\rho=\rho^{\prime}=0.5$. (The observed values are numerically reproducible to about \pm 0.00002 .) The agreement is strong. (Minor discrepancies will result from finite lattice size and sampling effects.)

Lastly, recall [Fig. 6(b)] that, for certain ranges of parameter values, there appear pronounced dips in the $f_{\text {rms }}$ plots before the asymptotic plateauing behavior emerges. At first glance this might seem surprising, but the origin of these dips is readily explained. For illustration, consider two asymmetrically coupled sandpiles, where sandpile $A$ is very weakly coupled to $A^{\prime}$ (i.e., $0<\gamma \ll 1$ ), and sandpile $A^{\prime}$ is even more weakly coupled to $A$ (i.e., take the extreme case where $\left.\gamma^{\prime}=0\right)$. Thus, when a site on $A$ topples, it has a very low probability of spilling grains to $A^{\prime}$ (during any single topple), and when a site on $A^{\prime}$ topples, it never spills grains onto $A$. Now suppose we drop grains onto sandpile $A$ only. Any small avalanches that result will almost always be entirely confined to $A$, in which case $f_{\text {rms }}$ [Eq. (1)] will approximately equal unity. On the other hand, for very large avalanches, most of the toppling sites will be on $A^{\prime}$, not $A$ (since $A^{\prime}$ receives from, but never spills back to, $A$ ). $f_{\text {rms }}$ will again approach unity for this case as well. Now, there must be some intermediate size avalanches for which the number of toppling sites on each sandpile is approximately equal; for this intermediate case $f_{\text {rms }}$ will be approximately zero. Hence, the overall shape of the $f_{\text {rms }}$ plot as a function of 
avalanche size will drop to zero somewhere in the middle. All of this presumes that the initial grains were dropped onto sandpile $A$ exclusively. Had the initial drops been onto sandpile $A^{\prime}$ instead, then no dips would have been seen, since $f_{\text {rms }}$ would have been pinned at unity for all avalanche sizes (since $\gamma^{\prime}=0$ ). In our actual simulations, the initial drop site was chosen randomly from among both sandpiles. Hence we would expect to see a partial dip (not all the way to zero) in the $f_{\text {rms }}$ curve, as was indeed the case [Fig. 6(b)].

\section{Onset of synchronization vs coupling strength}

In Sec. II C we described results from numerical simulations illustrating how variations in coupling strength affect the onset of synchronization for symmetrically coupled sandpiles (Fig. 5). Using the analytical results of Sec. III, we are now in a position to understand these numerical findings more fully.

We begin by noting that one approach to this problem would be to attempt to directly express the fractional deviation $f_{\text {rms }}$ as a function of coupling strength. This proves to be difficult for the following reason. From the definition of the two-point correlation functions $P(i, A), P\left(i, A^{\prime}\right), P^{\prime}\left(i, A^{\prime}\right)$, $P^{\prime}(i, A)$ of Sec. III D, it follows that the expected number of grains emerging from a site on sandpile $A$ due to the addition of a single grain to a site $i$ units to the left on $A$ is just $P(i, A) n_{A}$, while the expected number of grains emerging from the corresponding site on $A^{\prime}$ is $P\left(i, A^{\prime}\right) n_{A^{\prime}}$. (Analogous quantities can be defined if the initial grain is instead dropped onto sandpile $A^{\prime}$.) By summing up the grain contributions from all affected sites, it might thus appear that the rms fractional deviation could be expressed as

$$
f_{\mathrm{rms}}=\sqrt{\frac{1}{2}\left(\frac{\sum_{i} P(i, A) n_{A}-\sum_{i} P\left(i, A^{\prime}\right) n_{A^{\prime}}}{\sum_{i} P(i, A) n_{A}+\sum_{i} P\left(i, A^{\prime}\right) n_{A^{\prime}}}\right)^{2}+\frac{1}{2}\left(\frac{\sum_{i} P^{\prime}(i, A) n_{A}-\sum_{i} P^{\prime}\left(i, A^{\prime}\right) n_{A^{\prime}}}{\sum_{i} P^{\prime}(i, A) n_{A}+\sum_{i} P^{\prime}\left(i, A^{\prime}\right) n_{A^{\prime}}}\right)^{2}}
$$

where the summation over $i$ runs up to some appropriately chosen cutoff value (i.e., since the sum should be over affected sites only). However, while the above expression does indeed qualitatively reproduce the $f_{\text {rms }}$ curves seen in simulations-including the plateauing behavior and the dips found for asymmetric-coupling case-it nonetheless fails to provide a quantitatively accurate description. The underlying reason is that the quantities $P(i, A), P\left(i, A^{\prime}\right), P^{\prime}\left(i, A^{\prime}\right)$, $P^{\prime}(i, A)$, representing the expected number of topplings at a particular site if a single grain is added elsewhere, do not take into account the correlations which exist between toppling sites during a given avalanche. (We mention that the existence of such correlations between the number of grains spilled by different sites during a given avalanche is not at odds with our earlier finding that the invariant distribution for the automata can be written as a product of the invariant distributions of the individual sites.) Hence an alternate approach is required.

Towards this end, we first observe that the two-point correlation functions $P(i, A), P\left(i, A^{\prime}\right), P^{\prime}\left(i, A^{\prime}\right), P^{\prime}(i, A)$ all decay as $\lambda^{i}$, according to Eq. (3). We thus make the plausible (though formally unproven) assumption that synchronization between the sandpiles will set in as the quantity $\lambda^{i}$ becomes sufficiently small. [Note: an explicit expression for $\lambda$ in terms of the underlying toppling probabilities is readily obtained by following the steps leading to Eq. (3). For the case of symmetrically coupled sandpiles $\left(\rho=\rho^{\prime}, \gamma=\gamma^{\prime}\right)$, this expression simplifies nicely to $\lambda=(1-\gamma) /(1+\gamma)$.] Setting $\lambda^{i}$ $=\epsilon$ (where $\epsilon \ll 1$ represents a small, but nonzero, parameter) and solving for $i$, we see that $i \sim-1 / \log (\lambda)$. Henceforth we refer to this $i$ value as $i_{\text {onset }}$, since it should be regarded as the characteristic length (i.e., number of sites) at which the two sandpiles first synchronize. For weakly, symmetrically coupled sandpiles, $\log (\lambda)$ scales like $\gamma$; hence it follows that in the limit of weak coupling, $i_{\text {onset }} \sim 1 / \gamma$.

Next, we must relate the characteristic length $i_{\text {onset }}$ to the characteristic size $N_{\text {onset }}$ of an avalanche at onset of synchronization ( $N_{\text {onset }}$ represents the total number of grains spilled). To do so, we recall our earlier finding (Sec. III C) which demonstrated that the avalanching process for a single sandpile could be viewed as a fair random walk; however, by renormalizing across the two sandpiles, the large-scale joint behavior is expected to be that of a fair random walk. Reformulating the problem as a fair random walk, we ask the following: if a random walker takes a total of $i_{\text {onset }}$ steps before returning (for the first time) to the origin, what is the total integrated area $(N)$ of the walker's position vs time plot? This is readily estimated (from well known properties of Brownian motion) as follows: given $i_{\text {onset }}$ steps in total, the walker's typical distance from the origin will scale like $\sqrt{i_{\text {onset }}}$. So the integrated area will scale as $N_{\text {onset }} \sim i_{\text {onset }}^{3 / 2}$. Combining this with the preceding result, we therefore predict that

$$
N_{\text {onset }} \sim \frac{1}{\gamma^{3 / 2}}
$$

This result agrees reasonably well with the numerical scaling exponent found in Sec. II C [see in particular Fig. 5(b) [17]].

\section{CONCLUSION}

In the present work we have provided a numerical survey of observations about the behavior of systems of interacting 
SOC automata, and have shown how many of the essential features of the emergent statistical synchrony between automata can be understood using a variety of analytical tools. We conclude this report by pointing out several features which our analysis has not adequately addressed, along with several interesting questions which remain, as of yet, unanswered.

First, it has previously been demonstrated (see Ref. [3]) that self-organized criticality is not in fact an essential feature of the automata models for the emergence of large-scale synchrony. Indeed, the introduction of dissipation into these automata models destroys the SOC but not the large-scale synchrony; determining the necessary and sufficient criteria for the appearance of large-scale synchrony in automata remains an open question. Second, although one can use renormalization arguments heuristically for certain portions of our analysis, we do not know of a satisfactory renormalization procedure for the probabilistic sandpiles studied here. In particular, certain parts of the techniques used in Refs. [3,18$20]$ are not especially well suited to these models and would need to be significantly modified. Third, our measures of synchrony between sandpiles have relied primarily on indicators such as two-point correlation functions and mean scalars such as the rms fractional deviation. A deeper understanding of the nature of synchronization involving higherorder correlations among sites, such as multipoint correlation functions or conditional expectations, would seem to be particularly valuable. In addition, it would be interesting to study other measures of synchrony, such as correlations and conditional expectations involving $N_{A}$ and $N_{B}$.
Lastly, our analysis leading to the scaling relation (6) relied on two-point correlation functions and a random walk analysis, and provided a simple and intuitive heuristic for understanding the system's behavior. As suggested by an anonymous referee, our independence result of Sec. III B (demonstrating that the invariant distribution for the system could be written as a product of invariant distributions for the individual sites) together with the Abelian nature of our model might be used as a starting point for a potentially more direct (and perhaps more rigorous) derivation of the scaling relation, as follows: Drop a grain on sandpile $A$ and allow the avalanche to complete on that sandpile, ignoring for the moment any spills onto the other sandpile. Then repeat this procedure on sandpile $A^{\prime}$ for each of the grains that had spilled from $A$ to $A^{\prime}$. Continue this process until it stops. Then one might apply a central limit theorem to compute the asymptotic spill probabilities. However, the analysis of this process is nontrivial, as this is a stochastic branching process wherein the number of random variables in the sum could depend on the realizations of those variables. In addition, as noted in Sec. III B, it is not clear that there are no temporal correlations among sequential avalanches, which would also induce correlations among the random variables in the sum. Thus one would need a generalization of the central limit theorem for the analysis, which is possible but nontrivial. Nonetheless, we think this approach is extremely promising despite these nontrivial complications.

\section{ACKNOWLEDGMENT}

This research was supported by Research Corporation.
[1] P. Bak, C. Tang, and K. Wiesenfeld, Phys. Rev. Lett. 59, 381 (1987); Phys. Rev. A 38, 364 (1988).

[2] P. Bak and K. Chen, Physica D 38, 5 (1984); K. Chen and P. Bak, Phys. Lett. A 140, 299 (1989); Z. Olami, H.J.S. Feder, and K. Christensen, Phys. Rev. Lett. 68, 1244 (1992); V.M. Vinokur, M.V. Feigelman, and V.B. Geshkenbein, ibid. 67, 915 (1991); P. Bak and K. Sneppen, ibid. 71, 4083 (1993).

[3] E.J. Friedman and A.S. Landsberg, Phys. Rev. E 63, 051303 (2001)

[4] We employ the notation $0<\gamma \ll 1$ to signify that we are considering an arbitrarily small but fixed value of the coupling parameter $\gamma$ (as opposed to an asymptotic limit $\gamma \rightarrow 0$ ).

[5] This one-dimensional model was solved by Maslov and Zhang [10]. Interestingly, its behavior is quite similar to that of the two-dimensional, directed, deterministic model of Dhar and Ramaswamy [11]. Extensions to higher dimensions are described in Ref. [12].

[6] Note that this power-law scaling regime has both a lower cutoff (in which local interaction rules become important) and an upper cutoff (where finite system-size effects dominate).

[7] We have also examined the possible dependency of the exponent $q$ on the choice of cutoff threshold $f_{\text {onset }}$. Numerical simulations show that whatever dependency might exist is, at best, rather weak [e.g., for any $f_{\text {onset }} \in(0.3,0.7), q$ remains in the narrow range $(1.40,1.42)]$ - a level of variation which is on par with the numerical uncertainties of the simulations.

[8] In addition to behaving like the Dhar and Ramaswamy model, one can show, by relating this model to Brownian motion (as discussed in Sec. III C), that its behavior is typical for directed one-dimensional models.

[9] More formally, the rms fractional deviation for an avalanche of total size $N$ is defined as

$$
f_{\mathrm{rms}}=\sqrt{\frac{1}{N^{2}} \Sigma_{N_{A}, N_{A^{\prime}}}\left(N_{A}-N_{A^{\prime}}\right)^{2} P\left(N_{A}, N_{A^{\prime}} \mid N_{A}+N_{A^{\prime}}=N\right)} \text {, }
$$

where $P\left(N_{A}, N_{A^{\prime}} \mid N_{A}+N_{A^{\prime}}=N\right)$ is the joint probability distribution function of $N_{A}, N_{A^{\prime}}$ conditioned on $N_{A}+N_{A^{\prime}}=N$.

[10] S. Maslov and Y.-C. Zhang, Phys. Rev. Lett. 75, 1550 (1995).

[11] D. Dhar and R. Ramaswamy, Phys. Rev. Lett. 63, 1659 (1989).

[12] M. Kloster, S. Maslov and C. Tang, Phys. Rev. E 63, 026111 (2001).

[13] D. Dhar, Phys. Rev. Lett. 64, 1613 (1990).

[14] Y.C. Zhang, Phys. Rev. Lett. 63, 470 (1989).

[15] Note that one can easily compute the dynamical exponents from this observation. In addition, one can compute the asymptotic behavior for dissipative models since dissipation merely adds drift to the Brownian motion. For example, such calculations agree with the numerical data in Ref. [16].

[16] P. Ghaffari, S. Lise, and H. Jensen, Phys. Rev. E 56, 6702 (1997).

[17] Despite the approximate agreement with numerics, our derivation of the scaling exponent in Eq. (6) should not be regarded 
as a proof, for although it starts with an exact result (the twopoint correlation functions), it does utilize several arguments which are not rigorous.

[18] L. Pietronero, A. Vespignani, and S. Zapperi, Phys. Rev. Lett.
72, 1690 (1994).

[19] A. Vespignani, S. Zapperi, and L. Pietronero, Phys. Rev. E 51, 1711 (1995).

[20] J. Hasty and K. Wiesenfeld, Phys. Rev. Lett. 81, 1722 (1998). 